\title{
THE BISPECTRUM ANALYSIS TECHNIQUE IN MILLIMETER
} INTERFEROMETRY

\section{KOH-ICHIRO MORITA}

Nobeyama Radio Observatory, National Astronomical Observatory, Nobeyama, Minamimaki, Nagano, 384-13 JAPAN

\begin{abstract}
We propose an application of the bispectrum analysis technique in millimeter interferometry, which is used in the speckle masking technique. For the millimeter interferometry, in which atmospheric phase fluctuations are very serious, not only bispectrum phase but also bispectrum amplitude is good observable. We have developed an algorithm to reconstruct images directly from bispectrum. We have made good maps with this algorithm in the case of both simple and compact sources.
\end{abstract}

\section{INTRODUCTION}

At millimeter wavelengths, phase fluctuations due to atmospheric turbulence are large and the time scale for variability is very short so that, sometimes, it is very difficult to obtain a good image with a radio interferometer. At centimeter wavelengths, the phase closure imaging technique, as hybrid mapping or self-calibration (see e.g. Pearson and Readhead 1984), is very powerfull to cancel phase fluctuations for bright sources. In most cases of millimeter interferometry, however, it is difficult to obtain good signal to noise ratios and, therefore, it is necessary to develop an effective imaging algorithm for weak sources under the condition of large phase fluctuations.

Bispectrum is originally used by the speckle masking technique, which is one of the promising methods to obtain diffraction limited images in optical astronomy (Lohmann et al. 1983 ). For radio interferometry, bispectrum is given as:

$$
b_{i j k}(t)=V_{i j}(t) V_{j k}(t) V_{i k}^{*}(t)
$$

$V:$ Visibility.

$i, j, k$ : Antenna number.

Phase part of bispectrum is equal to closure phase. Cornwell(1987) showed that the bias in closure phase for poor signal to noise ratios can be removed by averaging the bispectrum and then taking the phase part. 

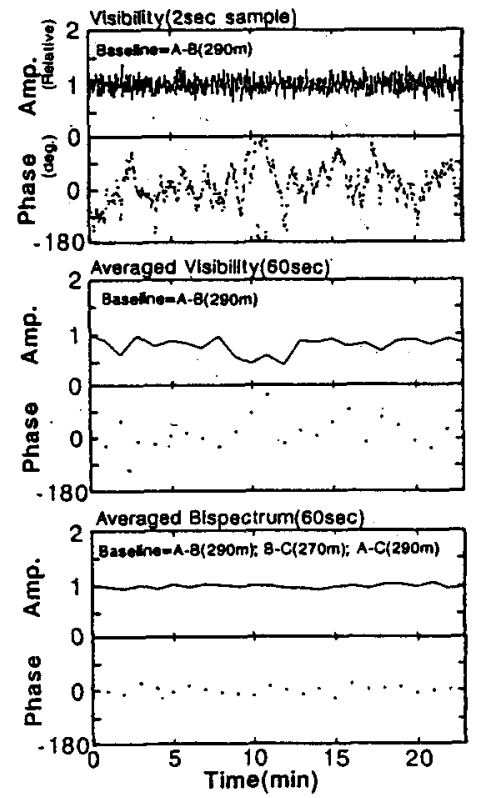

Fig. 1. Visibility and bispectrum observed with the NMA for $3 \mathrm{C} 84$ at $87 \mathrm{GHz}$.

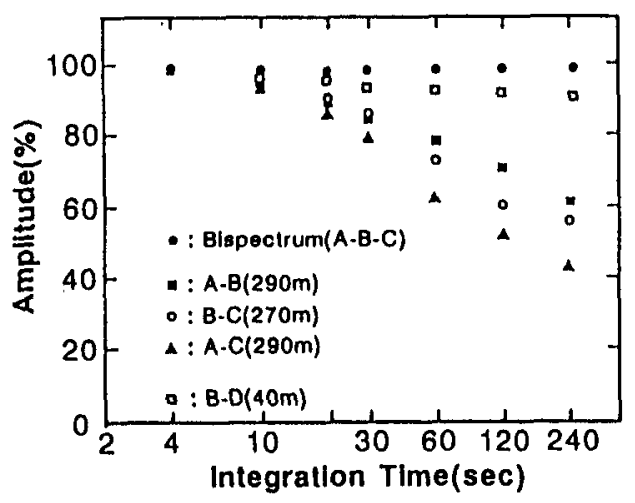

Fig. 2. Amplitude degradation.

\section{ADVANTAGE OF BISPECTRUM AMPLITUDE}

Fig. 1 shows an example of observations with the Nobeyama Millimeter Array(NMA). Large phase flucuations due to atmospheric turbulence are seen in visibility data, but are canceled out in averaged bispectrum. The time scales of phase fluctuations are very short $(<100 \mathrm{sec})$ and cause not only phase errors but also amplitude errors in the averaged visibility. In Fig. 2, we show the amplitude degradation in the averaged visibility and the averaged bispectrum. The amplitude degradation in the averaged visibility at short baseline is not so large, while it is very serious at longer baseline $(>200 \mathrm{~m})$. Thus it is obvious that bispectrum amplitude is also good observable in millimeter interferometry.

\section{AN IMAGING ALGORIGTHM FROM BISPECTRUM DATA}

In this section, we propose an imaging algorithm using both amplitude and phase of bispectrum. The algorithm is based on model fitting with a set of point sources like the CLEAN technique. In this algorithm, we fit a point source succesively in 3-dimensional grid points (position and intensity). In the $n$th step of the algorithm, distances between the observed bispectrum and the calculated bispectrum from a source model are made at each grid point. The flow chart for the algorithm is shown in Fig. 3. 


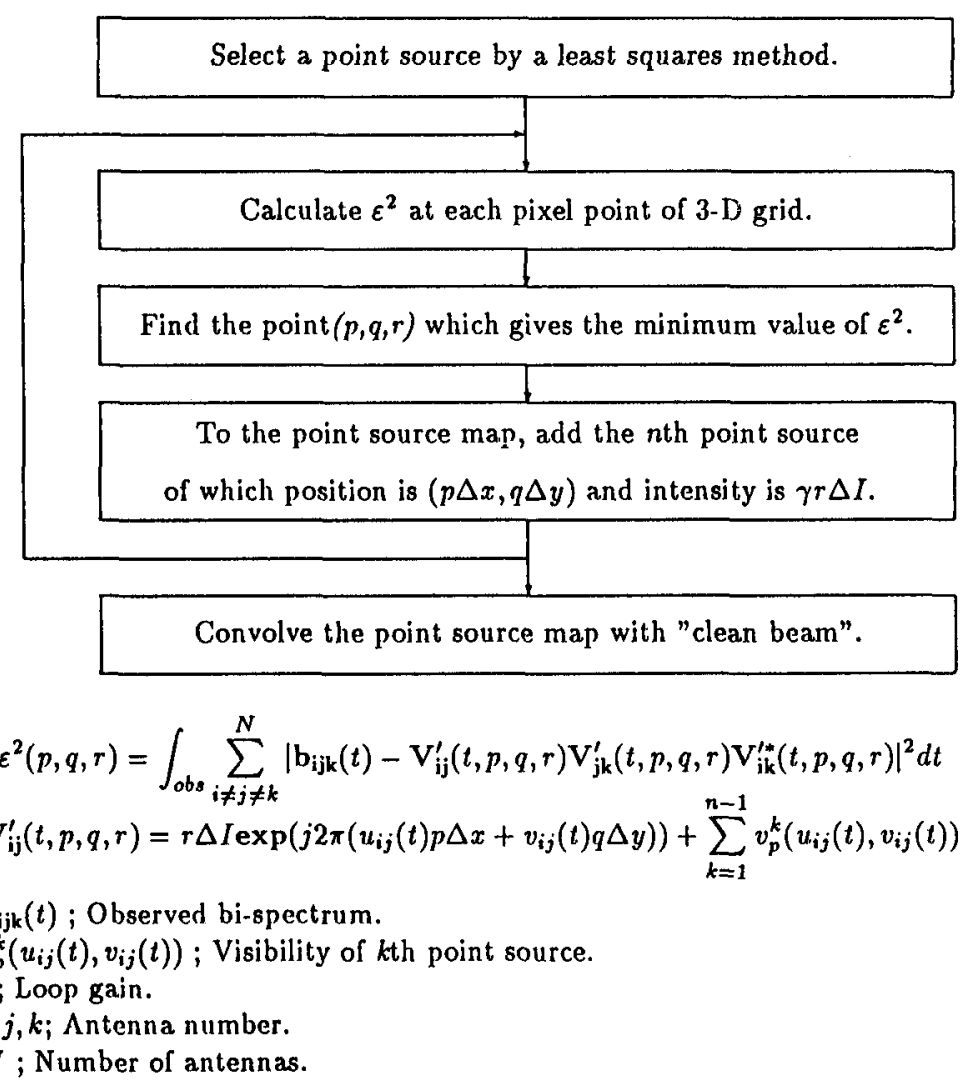

Fig. 3. The flow chart of the imaging algorithm.

\section{TESTS OF THE IMAGING ALGORITHM}

We compare our imaging algorithm with standard phase closure techniques (self-calibration, hybrid mapping). For the phase fluctuation, we used seeing monitor data at $19.45 \mathrm{GHz}$ at the NMA site (Ishiguro et al. 1991), being scaled at $230 \mathrm{GHz}$. We assumed that the array configuration is equal to the B-array of the NMA, which was almost similar configuration to that of the seeing monitor system. The observing duration was assumed to be 8 hours.

In Fig. 4, we show an example of imaging simulations. Fig. 4 (a) is the source model, which consists of two point sources and a gaussian source. In this simulation, night time seeing data in March 1990 was used as the phase fluctuation reference. We added thermal noise to visibility data so that the typical signal to noise ratio in a coherent integration time ( $30 \mathrm{sec})$ ranges from 3 - 10. The image of Fig. 4 (d) which is obtained with our algorithm is superior to those of (b) and (c). Fig. 4 (b) and (c) are results of standard phase closure techniques, in which two point sources are slightly extended and the gaussian 

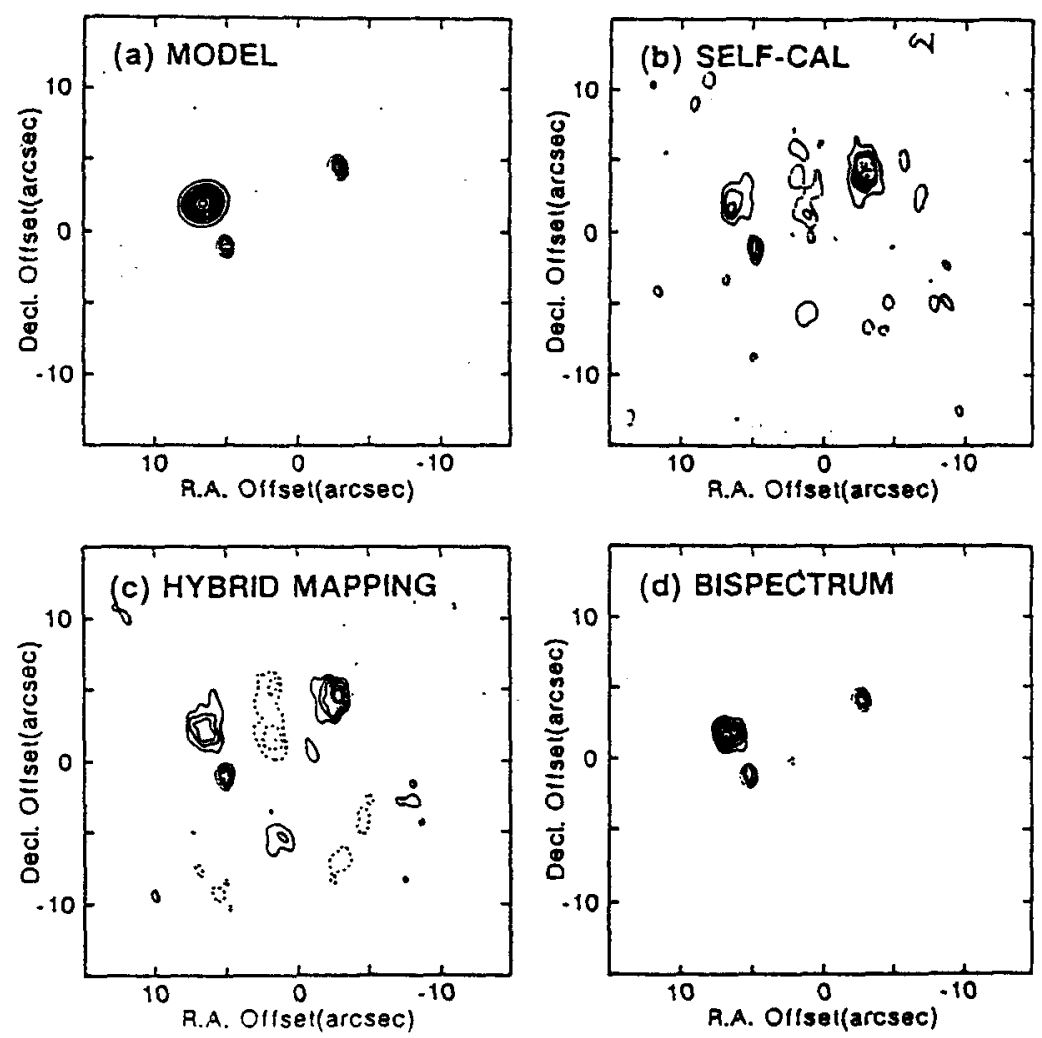

Fig. 4. Results of the imaging simulation. The contour interval is $5 \%$ of the peak level of each figure.

source is too weak. Therefore, in the case that the mapping ob ject is simple and compact, we can make a good map with this imaging algorithm.

For more extended source models, we failed to obtain good images with the algorithm. We suspect that there is some problem in the initial model and it will be necessary to improve the initial step of the algorithm.

\section{REFERENCES}

Cornwell, T. J. 1987, Astr. Ap., 180, 269.

Ishiguro, M., Kanzawa, T., and Kasuga, T. 1991, in this proceeding.

Lohmann, A. W., Weigelt, G., and Wirnitzer, B. 1983, Applied Optics, 22, 4028.

Pearson, T. J., and Readhead, A. C. S. 1984, Ann. Rev. Astron. Astrophys., 22, 97. 
S. R. Kulkarni: As Tim Cornwell pointed out, in the low SNR regime the bispectrum algorithm is straightforward: use all bispectrum phasers. However in the high SNR case (SNR of single visibility $\geq 3$ ) there is tremendous cross-talk between pairs of bispectrum phasers (see Kulkarni, A. J. 1990). In that case, it is not clear to me how one should do the bispectrum filling. Thus, it would seem to me that in this case, it is better to use self-cal since its mathematical basis is simple and straight forward. Thus bispectrum imaging is probably useful only in the region $3 \geq S N R \geq 1$. 